\title{
QUADRATIC PROBLEMS WITH TWO QUADRATIC CONSTRAINTS: CONVEX QUADRATIC RELAXATION AND STRONG LAGRANGIAN DUALITY
}

\author{
Abdelouahed Hamdi ${ }^{1, *}$, Akram TaAti $^{2}$ And Temadher A. Almaadeed ${ }^{1}$
}

\begin{abstract}
In this paper, we study a nonconvex quadratic minimization problem with two quadratic constraints, one of which being convex. We introduce two convex quadratic relaxations (CQRs) and discuss cases, where the problem is equivalent to exactly one of the CQRs. Particularly, we show that the global optimal solution can be recovered from an optimal solution of the CQRs. Through this equivalence, we introduce new conditions under which the problem enjoys strong Lagrangian duality, generalizing the recent condition in the literature. Finally, under the new conditions, we present necessary and sufficient conditions for global optimality of the problem.
\end{abstract}

Mathematics Subject Classification. 90C20, 90C22, 90C26, 90C42.

Received August 7, 2020. Accepted November 13, 2020.

\section{INTRODUCTION}

Consider the following nonconvex quadratic optimization problem:

$$
\begin{aligned}
p^{*}:=\inf & q_{1}(x):=x^{T} A x+2 a^{T} x \\
\text { s.t. } & q_{2}(x):=x^{T} B x+2 b^{T} x+\beta \leq 0, \\
& q_{3}(x):=x^{T} C x+2 d^{T} x+\gamma \leq 0,
\end{aligned}
$$

where $A, B, C \in \mathbb{R}^{n \times n}$ are symmetric matrices, $a, b, d \in \mathbb{R}^{n}$ and $\beta, \gamma \in \mathbb{R}$. In this paper, we study the case that matrix $C$ is positive semidefinite. Model problem $(\mathrm{P})$ arises in many areas such as constrained and unconstrained nonlinear optimization problems when the trust-region methods are applied to solve [13,15], double well potential problems [16], solving an inverse problem via regularization [17,19], the numerical solution of parameter identification problems [5,36], the robust formulation of convex quadratic inequalities with ellipsoidal implementation errors [7]. More applications can be found, e.g., in [7] (Rem. 2.5 and Sect. 3). The trust-region subproblem (TRS):

$$
\begin{aligned}
\min & x^{T} A x+2 a^{T} x \\
\text { s.t. } & \|x\| \leq \Delta,
\end{aligned}
$$

Keywords. Quadratically constrained quadratic programming, convex quadratic relaxation, strong duality, SDO-relaxation.

1 Department of Mathematics, Statistics, and Physics, Qatar University, Doha, Qatar.

2 Faculty of Mathematical Sciences, University of Guilan, Rasht, Iran.

*Corresponding author: abhamdi@qu.edu.qa, whamdi99@gmail.com 
is a special case of $(\mathrm{P})$ that is an essential subproblem in trust-region methods for nonlinear optimization [15]. It is well-known that TRS can be solved efficiently both in theory and practice $[2,15,18,20,35,39]$. Most importantly, TRS enjoys many useful and attractive features such as strong Lagrangian duality and exact semidefinite optimization (SDO) relaxation $[18,48]$. Problem (P) with one general quadratic inequality constraint, known as the generalized trust-region subproblem (GTRS), has been widely studied in the literature. Several methods have been derived for solving GTRS under various assumptions $[1,7,8,16,29,30,32,34,37,41,45,46,48]$. It has strong duality and an exact SDO-relaxation under the Slater condition [37,48]. Ben-Tal and den Hertog [7] have derived a second order cone programming reformulation for GTRS under a simultaneously diagonalizability assumption. Most recently, in [29], the authors have introduced a new convex quadratic reformulation for GTRS that minimizes a linear function subject to one or two convex quadratic constraints. They also have shown that the optimal solution of GTRS can be recovered from an optimal solution of the given reformulation.

The extended trust-region subproblem (eTRS) that enforces additional linear inequality constraints on the trust-region has been discussed in the literature [10,22,25-27,33,42-44]. Jeyakumar and Li [27] proved exactness of the SDO-relaxation for eTRS under a dimension condition. Moreover, they proved that the dimension condition together with the Slater condition ensure that strong Lagrangian duality holds for eTRS [27]. In the case of one linear constraint that is a special case of problem $(\mathrm{P})$, the dimension condition requires that the multiplicity of the minimum eigenvalue of the matrix $A$ must be at least 2 . The dimension condition is also extended to the trust-region subproblem with additional uniform convex quadratic inequality constraints [27]. Later, in [23], the authors improved the dimension condition for tightness of the SDO-relaxation of eTRS. Most recently, in [22], the authors have examined variants of TRS having additional conic constraint. They derived an exact convex relaxation under a structural condition on the conic constraint.

When $B=I, b=0$ and $C \succeq 0$, problem (P) reduces to the well-known Celis-Dennis-Tapia (CDT) problem. The CDT problem appears as a subproblem in some trust-region algorithms for constrained optimization where the original problem is to minimize a general nonlinear function subject to equality constraints [13,38]. Several articles have examined CDT and related problems [4,6,7,9,11,14,40,49-51]. In [11], the authors proved necessary and sufficient conditions for local and global optimality of the CDT problem via copositivity that gives a complete characterization in the degenerate case. Problem (P) with indefinite $B$ and positive definite $C$ has been studied in $[4,40]$. In [4], the authors presented a necessary and sufficient condition based on an optimal solution of the SDO-relaxation to characterize when the problem and its Lagrangian dual admit no duality gap. Moreover, they showed that if strong duality holds then an optimal solution can be obtained from an optimal solution of the SDO-relaxation, by means of a matrix rank-one decomposition procedure. In [40], the authors introduced a polynomial-time algorithm that computes all KKT points by solving a two-parameter linear eigenvalue problem. Then, the algorithm finds a global optimal solution as the KKT point with the smallest objective value. A geometric condition ensuring exact copositive relaxation for problem $(\mathrm{P})$ with indefinite $B$, positive semidefinite $C$ and additional linear inequality constraints is given in [12]. In [7,32], the authors derived a second order cone programming (SOCP) relaxation for problem (P) where the quadratic forms are simultaneously diagonalizable. They proved that if certain additional conditions hold, then the optimal solution of problem $(\mathrm{P})$ can be obtained from the optimal solution of the SOCP relaxation [7]. However, they also illustrated that the SOCP relaxation may not return the optimal solution of $(\mathrm{P})$ even when $B \succ 0$ and the second constraint is a linear inequality.

In this paper, we consider problem $(\mathrm{P})$ where $C \succeq 0$. We also assume that there exists $\hat{\lambda} \geq 0$ such that $A+\hat{\lambda} B \succ 0$. Obviously, this problem is more general than the CDT problem since the first constraint can be nonconvex. Motivated by [29], we present two convex quadratic relaxations (CQRs) under two different conditions for problem $(\mathrm{P})$. The CQRs minimize a linear objective function over three convex quadratic constraints. In the case of GTRS, our CQRs reduce to the ones proposed in [29]. Then we discuss when the CQRs are exact and return an optimal solution to problem (P). This results in sufficient conditions based on one (any) optimal solution of CQRs under which problem $(\mathrm{P})$ is equivalent to exactly one of the CQRs, and the optimal solution of $(\mathrm{P})$ can be recovered from an optimal solution of the CQRs. We also show that these conditions are sufficient for strong Lagrangian duality and consequently for exactness of the SDO-relaxation of (P). Although a direct 
verification of the sufficient conditions requires an optimal solution of the CQRs, it is possible to use them in order to find the ones which are expressed in terms of the data of the problem. These conditions are in fact an extension of the one given in [22] for the CDT problem as a special case to more general model problem (P). The sufficient conditions introduced in the paper, ensure the exactness of both the CQRs and the SDO-relaxation of problem $(\mathrm{P})$. It is worth noting that the solution of a semidefinite problem is still an intractable task for many practical large-scale or dense problems, while the CQRs are significantly more tractable than SDOs, and advanced commercial software is available to solve them [21]. Finally, as a consequence, we present necessary and sufficient conditions for global optimality of problem $(\mathrm{P})$ under the new conditions together with the Slater condition.

As mentioned before, a necessary and sufficient condition for strong Lagrangian duality of problem (P) where $C \succ 0$ is given in [4]. The condition requires an optimal solution of the SDO-relaxation and hence can not be verified for large-scale instances. In contrast, both of our sufficient conditions, the one based on an optimal solution of the CQRs and the other based on the data of the problem, can be verified efficiently for large-scale instances. From a convex reformulation perspective, both the SOCP relaxation studied in $[7,32]$ and our CQRs are convex quadratic problems and significantly more tractable than the SDO-relaxation. It should be noted that the former covers instances of problem $(\mathrm{P})$ where the Hessian of the quadratic forms are simultaneously diagonalizable, but, in general, this property does not hold for the underlying problem in the paper. Conditions for simultaneous diagonalizability and corresponding algorithms are investigated recently in [28]. Most recently, in [47], it has been shown that under the simultaneously diagonalizability assumption, the SDO-relaxation is in fact equivalent to the SOCP relaxation from [7,32]. Therefore, when the simultaneously diagonalizability assumption and the given sufficient conditions in the paper hold, the CQRs and the SOCP relaxation are equivalent.

The rest of the paper is organized as follows. In Section 2, we introduce the CQRs. Then, in Section 3, we discuss when and how one can obtain an optimal solution of problem $(\mathrm{P})$ from an optimal solution of the CQRs, revealing new sufficient conditions for strong Lagrangian duality of problem (P). In Section 4, we give sufficient conditions for exactness of the CQRs and strong Lagrangian duality of problem (P) in terms of the data of the problem and then compare them with the related conditions from the literature. We also present necessary and sufficient conditions for global optimality of problem (P).

Notations. Throughout this paper, for a symmetric matrix $A, A \succ 0(A \succeq 0)$ denotes $A$ is positive definite (positive semidefinite). Moreover, $\operatorname{Null}(A)$ and $\operatorname{Rank}(A)$ denote its Null space and Rank. For two symmetric matrices $A$ and $B$, we use $\lambda_{\min }(A, B)$ to denote the smallest generalized eigenvalue of the pencil $A-\lambda B$ and $\lambda_{\min }(A)$ to denote the smallest eigenvalue of matrix $A$. Finally, $A \bullet B:=\operatorname{trace}(A B)$ is the usual matrix inner product of two symmetric matrices $A$ and $B$.

\section{Convex quadratic Relaxations}

In this section, we present two new CQRs for problem (P) under two different conditions. These CQRs are later used to solve problem $(\mathrm{P})$ globally and to derive sufficient conditions under which problem $(\mathrm{P})$ enjoys strong Lagrangian duality and exact SDO-relaxation. We assume that $C$ is positive semidefinite and also consider the following assumptions.

Assumption 2.1. There exists $\hat{\lambda} \geq 0$ such that $A+\hat{\lambda} B \succ 0$.

Assumption 2.2. The Slater condition holds for problem $(P)$, i.e., there exists $\hat{x}$ with $q_{2}(\hat{x})<0$ and $q_{3}(\hat{x})<0$.

Under Assumptions 2.1 and 2.2, problem $(\mathrm{P})$ is solvable due to the following lemma.

Lemma 2.3. Suppose that Assumptions 2.1 and 2.2 hold. Then problem $(P)$ is solvable, i.e., the infimum in $(P)$ is always attainable. 
Proof. It is obvious that problem $(\mathrm{P})$ is equivalent to its epigraph reformulation as follows:

$$
\begin{array}{rl}
p^{*}=\inf _{t, x} & t \\
\text { s.t. } & q_{1}(x) \leq t, \\
& q_{2}(x) \leq 0, \\
& q_{3}(x) \leq 0 .
\end{array}
$$

Under Assumptions 2.1 and 2.2, it is well-known that problem (P) without the second constraint is bounded from below (see [24], Thm. 5). This implies that $\left(P_{0}\right)$ is equivalent to the following problem:

$$
\begin{array}{rl}
p^{*}=\inf _{t, x} & t \\
\text { s.t. } & q_{1}(x) \leq t, \\
& q_{2}(x) \leq 0, \\
& q_{3}(x) \leq 0, \\
& \tilde{t} \leq t \leq M,
\end{array}
$$

where $\tilde{t}$ is the optimal (infimum) value of $q_{1}(x)$ over the constraint $q_{2}(x) \leq 0$ and $M$ is a sufficiently large constant. Let $S$ denote the feasible region of problem (2.1). The set $S$ is closed and the objective function in problem (2.1) is continuous. Therefore, to prove the assertion, it is sufficient to establish that $S$ is bounded. Since $\tilde{t} \leq t \leq M$, we only need to show that there exists $\hat{M}>0$ such that $\|x\| \leq \hat{M}$ for all $(x, t) \in S$. To do so, let $h(x):=q_{1}(x)+\hat{\lambda} q_{2}(x)$ where $\hat{\lambda}$ is the same as in Assumption 2.1. The function $h$ is strictly convex and for any $(x, t) \in S$, we have $h(x) \leq M$ which completes the proof.

By Assumption 2.1, matrices $A$ and $B$ are simultaneously diagonalizable by congruence [31], i.e., there exists an invertible matrix $Q$ and diagonal matrices $D=\operatorname{diag}\left(\alpha_{1}, \ldots, \alpha_{n}\right)$ and $E=\operatorname{diag}\left(e_{1}, \ldots, e_{n}\right)$ such that $Q^{T} A Q=D$ and $Q^{T} B Q=E$. It is easy to verify that $A+\lambda B \succeq 0$ if and only if $\lambda \in\left[\lambda_{1}, \lambda_{2}\right]$ where

$$
\lambda_{1}=\max \left\{-\frac{\alpha_{i}}{e_{i}} \mid e_{i}>0\right\}, \quad \lambda_{2}=\min \left\{-\frac{\alpha_{i}}{e_{i}} \mid e_{i}<0\right\} .
$$

If $A \succ 0, \lambda_{1}<0$ else $\lambda_{1} \geq 0$. We have two cases for the set $I_{\mathrm{PSD}}=\{\lambda \geq 0 \mid A+\lambda B \succeq 0\}$ as follows, where $\hat{\lambda}_{1}=\max \left\{0, \lambda_{1}\right\}$ :

Condition 1. $I_{\mathrm{PSD}}=\left[\hat{\lambda}_{1}, \lambda_{2}\right]$ if $B$ is not positive semidefinite.

Condition 2. $I_{\mathrm{PSD}}=\left[\hat{\lambda}_{1}, \infty\right)$ if $B$ is positive semidefinite.

Note that, in Condition 1 , the interval $\left[\hat{\lambda}_{1}, \lambda_{2}\right]$ is not a singleton, i.e., $\hat{\lambda}_{1}<\lambda_{2}$. To see this, first let $\hat{\lambda}_{1}=\lambda_{1}$. By Assumption 2.1, the open interval $\left(\lambda_{1}, \lambda_{2}\right)$ is nonempty. Therefore, $\hat{\lambda}_{1}<\lambda_{2}$. Next, let $\hat{\lambda}_{1}=0$. Then $\lambda_{1}<0$ and consequently $A \succ 0$. This implies that $D \succ 0$ and thus $\alpha_{i}>0$ for all $i=1, \ldots, n$. Therefore, by the definition of $\lambda_{2}$, we have $\lambda_{2}>0$, implying that $\hat{\lambda}_{1}<\lambda_{2}$.

Now we are ready to introduce two new CQRs $\left(P_{1}\right)$ and $\left(P_{2}\right)$ corresponding to Conditions 1 and 2, respectively, by defining $h_{1}(x)=q_{1}(x)+\hat{\lambda}_{1} q_{2}(x)$ and $h_{2}(x)=q_{1}(x)+\lambda_{2} q_{2}(x)$ :

$$
\begin{array}{rl}
p_{1}^{*}:=\inf _{x, t} & t \\
\text { s.t. } & h_{1}(x) \leq t, \\
& h_{2}(x) \leq t, \\
& q_{3}(x) \leq 0,
\end{array}
$$


and

$$
\begin{aligned}
p_{2}^{*}:=\inf _{x} & h_{1}(x) \\
\text { s.t. } & q_{2}(x) \leq 0, \\
& q_{3}(x) \leq 0 .
\end{aligned}
$$

Recall that we assumed $C$ is positive semidefinite. When both $A$ and $B$ are positive semidefinite, then Condition 2 holds with $\hat{\lambda}_{1}=0$ and thus problem $\left(P_{2}\right)$ reduces to problem $(\mathrm{P})$ which is already a convex quadratic problem. Hence, from now on, we assume that at least one of $q_{1}(x)$ and $q_{2}(x)$ is nonconvex. Problems $\left(P_{1}\right)$ and $\left(P_{2}\right)$ are both convex. Suppose that Condition 1 holds and $x$ is a feasible solution of $\left(P_{0}\right)$. Since $q_{2}(x) \leq 0$ and $\hat{\lambda}_{1}, \lambda_{2} \geq 0$, then

$$
\begin{aligned}
& h_{1}(x)=q_{1}(x)+\hat{\lambda}_{1} q_{2}(x) \leq q_{1}(x) \leq t, \\
& h_{2}(x)=q_{1}(x)+\lambda_{2} q_{2}(x) \leq q_{1}(x) \leq t .
\end{aligned}
$$

Therefore, the feasible region of $\left(P_{1}\right)$ contains that of $\left(P_{0}\right)$ and since the two problems have the same objective function, then we have $p_{1}^{*} \leq p^{*}$, i.e., $\left(P_{1}\right)$ is a convex relaxation of $\left(P_{0}\right)$. Next, suppose that Condition 2 holds. Problems $\left(P_{2}\right)$ and $(\mathrm{P})$ have the same feasible region and since $h_{1}(x) \leq q_{1}(x)$, we have $p_{2}^{*} \leq p^{*}$, implying that $\left(P_{2}\right)$ is a convex relaxation of $(\mathrm{P})$. The following lemma states that problems $\left(P_{1}\right)$ and $\left(P_{2}\right)$ are bounded from below and their optimal values are attained.

Lemma 2.4. Under Assumptions 2.1 and 2.2, problems $\left(P_{1}\right)$ and $\left(P_{2}\right)$ are bounded from below and their optimal values are attained.

Proof. Consider problem $\left(P_{1}\right)$. By Theorem 2.9 of [29], problem

$$
\begin{array}{ll}
\inf _{x, t} & t \\
\text { s.t. } & h_{1}(x) \leq t, \\
& h_{2}(x) \leq t,
\end{array}
$$

is bounded from below and its optimal value is attained. This implies that problem $\left(P_{1}\right)$ is equivalent to the following problem:

$$
\begin{array}{rl}
p_{1}^{*}=\inf _{x, t} & t \\
\text { s.t. } & h_{1}(x) \leq t, \\
& h_{2}(x) \leq t, \\
& q_{3}(x) \leq 0, \\
& \hat{t} \leq t \leq M,
\end{array}
$$

where $\hat{t}$ is the optimal value of (2.2) and $M$ is a sufficiently large constant. Let $S$ denote the feasible region of (2.3). The set $S$ is closed and the objective function in (2.3) is continuous. Therefore, to prove the assertion, it is sufficient to establish that $S$ is bounded. Since $\hat{t} \leq t \leq M$, we only need to show there exists $\hat{M}>0$ such that $\|x\| \leq \hat{M}$ for all $(x, t) \in S$. To this end, let $h(x):=\alpha_{1} h_{1}(x)+\alpha_{2} h_{2}(x)$ where $\alpha_{1}, \alpha_{2}>0$ and $\alpha_{1}+\alpha_{2}=1$. The function $h$ is strictly convex and for any $(x, t) \in S$, we have $h(x) \leq M$. The proof for problem $\left(P_{2}\right)$ is similar.

Remark 2.5. Recall that we have assumed that there exists $\hat{\lambda} \geq 0$ such that $A+\hat{\lambda} B \succ 0$. Then, $\lambda_{1}$ and $\lambda_{2}$ can be efficiently computed via finding some generalized eigenvalues of a matrix pencil, see [29,37,45]. Precisely, $A+\lambda B \succeq 0$ if and only if $\lambda_{1} \leq \lambda \leq \lambda_{2}$ where $\lambda_{1}=\underline{\lambda}+\hat{\lambda}, \lambda_{2}=\bar{\lambda}+\hat{\lambda}$,

$$
\underline{\lambda}= \begin{cases}\frac{1}{\lambda_{\min }(-B, A+\hat{\lambda} B)} & \text { if } \lambda_{\min }(-B, A+\hat{\lambda} B)<0, \\ -\infty & \text { otherwise }\end{cases}
$$


and

$$
\bar{\lambda}= \begin{cases}\frac{1}{\lambda_{\min }(B, A+\hat{\lambda} B)} & \text { if } \lambda_{\min }(B, A+\hat{\lambda} B)<0, \\ \infty & \text { otherwise. }\end{cases}
$$

\section{Global minimization And Strong DUALity}

Here, we discuss cases where the nonconvex problem $(\mathrm{P})$ is equivalent to one of the CQRs $\left(P_{1}\right)$ and $\left(P_{2}\right)$, i.e., $p^{*}=p_{1}^{*}$ or $p^{*}=p_{2}^{*}$ and the optimal solution of $(\mathrm{P})$ can be recovered from an optimal solution of the CQRs. Moreover, through this equivalence, new conditions under which problem $(\mathrm{P})$ enjoys strong Lagrangian duality are introduced.

The following theorem states a necessary and sufficient condition for the exactness of the CQRs $\left(P_{1}\right)$ and $\left(P_{2}\right)$.

Theorem 3.1. Suppose that Assumptions 2.1 and 2.2 hold.

(i) Under Condition 1, problem $(P)$ is equivalent to the $C Q R\left(P_{1}\right)$, i.e., $p^{*}=p_{1}^{*}$ if and only if there exists an optimal solution $\left(x^{*}, t^{*}\right)$ to problem $\left(P_{1}\right)$ such that $h_{1}\left(x^{*}\right)=h_{2}\left(x^{*}\right)=t^{*}$ or $h_{2}\left(x^{*}\right)<t^{*}$ and $\hat{\lambda}_{1}=0$. Moreover, $x^{*}$ is optimal for problem $(P)$.

(ii) Under Condition 2, problem $(P)$ is equivalent to the $C Q R\left(P_{2}\right)$, i.e., $p^{*}=p_{2}^{*}$ if and only if there exists an optimal solution $x^{*}$ to problem $\left(P_{2}\right)$ such that $q_{2}\left(x^{*}\right)=0$. Moreover, $x^{*}$ is optimal for problem $(P)$.

Proof. (i) First note that for any optimal solution $\left(x^{*}, t^{*}\right)$ of problem $\left(P_{1}\right)$, we have $h_{1}\left(x^{*}\right)=t^{*}$ or $h_{2}\left(x^{*}\right)=t^{*}$. To see this, suppose by contradiction that $h_{1}\left(x^{*}\right)<t^{*}$ and $h_{2}\left(x^{*}\right)<t^{*}$. Set $\tilde{t}=\max \left\{h_{1}\left(x^{*}\right), h_{2}\left(x^{*}\right)\right\}$. Then $\tilde{t}<t^{*}$ and $\left(x^{*}, \tilde{t}\right)$ is a feasible solution of $\left(P_{1}\right)$ with smaller objective value than $t^{*}$. This contradicts the fact that $\left(x^{*}, t^{*}\right)$ is an optimal solution of $\left(P_{1}\right)$. Now suppose that there exists an optimal solution $\left(x^{*}, t^{*}\right)$ to problem $\left(P_{1}\right)$ such that $h_{1}\left(x^{*}\right)=h_{2}\left(x^{*}\right)=t^{*}$. Then $h_{1}\left(x^{*}\right)=h_{2}\left(x^{*}\right)=t^{*}$ implies that $\left(\hat{\lambda}_{1}-\lambda_{2}\right) q_{2}\left(x^{*}\right)=0$. Furthermore, since $\hat{\lambda}_{1} \neq \lambda_{2}$, we obtain $q_{2}\left(x^{*}\right)=0$, and consequently, $q_{1}\left(x^{*}\right)=h_{1}\left(x^{*}\right)-\hat{\lambda}_{1} q_{2}\left(x^{*}\right)=t^{*}$. These together with $q_{3}\left(x^{*}\right) \leq 0$ show that $\left(x^{*}, t^{*}\right)$ is also feasible for $\left(P_{0}\right)$ and since $\left(P_{1}\right)$ is a relaxation of $\left(P_{0}\right)$, then $\left(x^{*}, t^{*}\right)$ solves $\left(P_{0}\right), p^{*}=p_{1}^{*}$ and thus $x^{*}$ solves $(\mathrm{P})$. Next, suppose that there exists an optimal solution $\left(x^{*}, t^{*}\right)$ to problem $\left(P_{1}\right)$ such that $h_{2}\left(x^{*}\right)<t^{*}$ and $\hat{\lambda}_{1}=0$. Then, since $h_{2}\left(x^{*}\right)<t^{*}$, we have $h_{1}\left(x^{*}\right)=t^{*}$. Furthermore, $h_{2}\left(x^{*}\right)<t^{*}$ and $h_{1}\left(x^{*}\right)=t^{*}$ imply that $\left(\lambda_{2}-\hat{\lambda}_{1}\right) q_{2}\left(x^{*}\right)<0$. Then $\lambda_{2}>\hat{\lambda}_{1}$ results in $q_{2}\left(x^{*}\right)<0$. Moreover, we have $q_{1}\left(x^{*}\right)=h_{1}\left(x^{*}\right)-\hat{\lambda}_{1} q_{2}\left(x^{*}\right)=t^{*}$. These together with the fact that $q_{3}\left(x^{*}\right) \leq 0$ show that $\left(x^{*}, t^{*}\right)$ is also feasible for $\left(P_{0}\right)$ and since $\left(P_{1}\right)$ is a relaxation of $\left(P_{0}\right)$, then $\left(x^{*}, t^{*}\right)$ solves $\left(P_{0}\right), p^{*}=p_{1}^{*}$ and thus $x^{*}$ solves problem $(\mathrm{P})$. To prove the converse, we show that $p_{1}^{*}<p^{*}$ if for every optimal solution $\left(x^{*}, t^{*}\right)$ of problem $\left(P_{1}\right), h_{1}\left(x^{*}\right)<t^{*}$ or $h_{2}\left(x^{*}\right)<t^{*}$ with $\hat{\lambda}_{1}>0$. First suppose that $h_{1}\left(x^{*}\right)<t^{*}$ for every optimal solution $\left(x^{*}, t^{*}\right)$ of problem $\left(P_{1}\right)$. In this case, we have $h_{2}\left(x^{*}\right)=t^{*}$. Then $h_{1}\left(x^{*}\right)<t^{*}$ and $h_{2}\left(x^{*}\right)=t^{*}$ imply that $\left(\hat{\lambda}_{1}-\lambda_{2}\right) q_{2}\left(x^{*}\right)<0$. Since $\hat{\lambda}_{1}<\lambda_{2}$, we obtain

$$
q_{2}\left(x^{*}\right)>0 .
$$

Suppose by contradiction that $p_{1}^{*}=p^{*}$ and let $(\bar{x}, \bar{t})$ be an optimal solution of $\left(P_{0}\right)$. Then $(\bar{x}, \bar{t})$ is feasible for $\left(P_{1}\right)$ and $p_{1}^{*}=t^{*}=p^{*}=\bar{t}$. This implies that $(\bar{x}, \bar{t})$ is also optimal for $\left(P_{1}\right)$. Therefore, $q_{2}(\bar{x})>0$ that contradicts the fact that $(\bar{x}, \bar{t})$ is feasible for $\left(P_{0}\right)$. Next consider the case where $h_{2}\left(x^{*}\right)<t^{*}$ and $\hat{\lambda}_{1}>0$ for every optimal solution $\left(x^{*}, t^{*}\right)$ of $\left(P_{1}\right)$. To prove that $p_{1}^{*}<p^{*}$, suppose by contradiction that $p_{1}^{*}=p^{*}$. Let $(\bar{x}, \bar{t})$ be an optimal solution of $\left(P_{0}\right)$. Then, $(\bar{x}, \bar{t})$ is feasible for $\left(P_{1}\right)$ and $p_{1}^{*}=t^{*}=p^{*}=\bar{t}$. This implies that $(\bar{x}, \bar{t})$ is also optimal for $\left(P_{1}\right)$. Then, $h_{2}(\bar{x})<\bar{t}$ and consequently $h_{1}(\bar{x})=\bar{t}$. Therefore, $q_{1}(\bar{x})=h_{1}(\bar{x})-\hat{\lambda}_{1} q_{2}(\bar{x})>\bar{t}$ since $\hat{\lambda}_{1}>0$ and $q_{2}(\bar{x}) \leq 0$. Then $q_{1}(\bar{x})>\bar{t}$ contradicts the fact that $(\bar{x}, \bar{t})$ is feasible for $\left(P_{0}\right)$.

(ii) First note that since we have assumed that $q_{1}(x)$ and $q_{2}(x)$ are not both convex, then $\hat{\lambda}_{1}>0$. Suppose that there exists an optimal solution $x^{*}$ to problem $\left(P_{2}\right)$ such that $q_{2}\left(x^{*}\right)=0$. Since $q_{2}\left(x^{*}\right)=0$ and $\left(P_{2}\right)$ is a relaxation of $\left(P_{0}\right)$, then $q_{1}\left(x^{*}\right)=p_{2}^{*} \leq p^{*} \leq q_{1}\left(x^{*}\right)$ and consequently $p^{*}=p_{2}^{*}=q_{1}\left(x^{*}\right)$ and $x^{*}$ solves $(\mathrm{P})$. To 
prove the converse, we show that $p_{2}^{*}<p^{*}$ if for every optimal solution $x^{*}$ of problem $\left(P_{2}\right), q_{2}\left(x^{*}\right)<0$. To see this, suppose by contradiction that $p_{2}^{*}=p^{*}$ and let $\bar{x}$ be an optimal solution of $\left(P_{2}\right)$. Since $\bar{x}$ is feasible for $\left(P_{2}\right)$ and $p_{2}^{*}=p^{*}, \bar{x}$ is also optimal for $\left(P_{2}\right)$ and thus $q_{2}(\bar{x})<0$. On the other hand, $p_{2}^{*}=q_{1}(\bar{x})+\hat{\lambda}_{1} q_{2}(\bar{x})=$ $p^{*}=q_{1}(\bar{x})$. Since $\hat{\lambda}_{1}>0$, we obtain $q_{2}(\bar{x})=0$ that contradicts the fact that $q_{2}(\bar{x})<0$.

The following theorem gives a sufficient condition under which strong Lagrangian duality holds for problem $(\mathrm{P})$.

Theorem 3.2. Suppose that Assumptions 2.1 and 2.2 hold.

(i) Under Condition 1, if there exists an optimal solution $\left(x^{*}, t^{*}\right)$ to problem $\left(P_{1}\right)$ such that $h_{1}\left(x^{*}\right)=h_{2}\left(x^{*}\right)=$ $t^{*}$ or $h_{2}\left(x^{*}\right)<t^{*}$ and $\hat{\lambda}_{1}=0$, then strong duality holds for problem $(P)$ and its Lagrangian dual problem is solvable.

(ii) Under Condition 2, if there exists an optimal solution $x^{*}$ to problem $\left(P_{2}\right)$ such that $q_{2}\left(x^{*}\right)=0$, then strong duality holds for problem $(P)$ and its Lagrangian dual problem is solvable.

Proof. (i) Since problem $\left(P_{1}\right)$ is convex and satisfies the Slater condition, there exist nonnegative multipliers $\mu_{1}^{*}, \mu_{2}^{*}$ and $\mu_{3}^{*}$ such that

$$
\begin{aligned}
\left(A+\left(\mu_{1}^{*} \hat{\lambda}_{1}+\mu_{2}^{*} \lambda_{2}\right) B+\mu_{3}^{*} C\right) x^{*} & =-\left(a+\left(\mu_{1}^{*} \hat{\lambda}_{1}+\mu_{2}^{*} \lambda_{2}\right) b+\mu_{3}^{*} d\right), \\
\mu_{1}^{*}\left(h_{1}\left(x^{*}\right)-t^{*}\right) & =0, \\
\mu_{2}^{*}\left(h_{2}\left(x^{*}\right)-t^{*}\right) & =0 \\
\mu_{3}^{*} q_{3}\left(x^{*}\right) & =0 \\
\mu_{1}^{*}+\mu_{2}^{*} & =1, \\
h_{1}\left(x^{*}\right) & \leq t^{*}, \\
h_{2}\left(x^{*}\right) & \leq t^{*}, \\
q_{3}\left(x^{*}\right) & \leq 0 .
\end{aligned}
$$

First suppose that there exists an optimal solution $\left(x^{*}, t^{*}\right)$ to problem $\left(P_{1}\right)$ such that $h_{1}\left(x^{*}\right)=h_{2}\left(x^{*}\right)=t^{*}$. Then $h_{1}\left(x^{*}\right)=h_{2}\left(x^{*}\right)=t^{*}$ implies that $q_{2}\left(x^{*}\right)=0$. Set $\lambda^{*}=\mu_{1}^{*} \hat{\lambda}_{1}+\mu_{2}^{*} \lambda_{2}$. Since $\mu_{1}^{*} \geq 0, \mu_{2}^{*} \geq 0$ and $\mu_{1}^{*}+\mu_{2}^{*}=1$, then $\lambda^{*} \in\left[\hat{\lambda}_{1}, \lambda_{2}\right]$ and thus $A+\lambda^{*} B \succeq 0$. Furthermore, since $\mu_{3}^{*} \geq 0$ and $C \succeq 0$, we have

$$
A+\lambda^{*} B+\mu_{3}^{*} C \succeq 0 .
$$

Also, we have

$$
\begin{aligned}
p^{*} \geq d^{*}: & =\max _{\gamma_{1}, \gamma_{2} \geq 0} \min _{x}\left\{q_{1}(x)+\gamma_{1} q_{2}(x)+\gamma_{3} q_{3}(x)\right\} \\
& \geq \min _{x}\left\{q_{1}(x)+\lambda^{*} q_{2}(x)+\mu_{3}^{*} q_{3}(x)\right\} \\
& =q_{1}\left(x^{*}\right)+\lambda^{*} q_{2}\left(x^{*}\right)+\mu_{3}^{*} q_{3}\left(x^{*}\right) \\
& =q_{1}\left(x^{*}\right) \geq p^{*}
\end{aligned}
$$

where the first inequality follows from the weak duality property, the second equality follows from (3.2) and (3.10), the third equality follows from (3.5) and $q_{2}\left(x^{*}\right)=0$ and the last inequality follows from the feasibility of $x^{*}$. Therefore, we have $p^{*}=d^{*}$, i.e., strong duality holds for problem $(\mathrm{P})$ and the maximum in (3.11) is attained. Next consider the case where there exists an optimal solution of $(\mathrm{P})$ such that $h_{2}\left(x^{*}\right)<t^{*}$ and $\hat{\lambda}_{1}=0$. By setting $\lambda^{*}=0$, the same approach as above can be applied to show that strong duality holds for $(\mathrm{P})$ and the Lagrangian dual problem is solvable. 
(ii) Suppose that there exists an optimal solution to $\left(P_{2}\right)$ such that $q_{2}\left(x^{*}\right)=0$. Problem $\left(P_{2}\right)$ is convex, satisfies the Slater condition and by Lemma 2.4 is solvable. Therefore, there exist nonnegative multipliers $\mu_{1}^{*}$ and $\mu_{2}^{*}$ such that

$$
\begin{aligned}
\left(A+\left(\hat{\lambda}_{1}+\mu_{1}^{*}\right) B+\mu_{2}^{*} C\right) x^{*} & =-\left(a+\left(\mu_{1}^{*}+\hat{\lambda}_{1}\right) b+\mu_{2}^{*} d\right), \\
\mu_{1}^{*} q_{2}\left(x^{*}\right) & =0, \\
\mu_{2}^{*} q_{3}\left(x^{*}\right) & =0, \\
q_{2}\left(x^{*}\right) & \leq 0, \\
q_{3}\left(x^{*}\right) & \leq 0 .
\end{aligned}
$$

By setting $\lambda^{*}=\mu_{1}^{*}+\hat{\lambda}_{1}$, the same discussion as part (i) can be applied to show that strong duality holds for problem $(\mathrm{P})$ and the Lagrangian dual problem is solvable.

When the CQRs $\left(P_{1}\right)$ and $\left(P_{2}\right)$ have unique optimal solution, the conditions given in Theorems 3.1 and 3.2 can be verified easily. In contrast, when there are multiple optimal solutions to the CQRs, Theorems 3.1 and 3.2 require scanning the set of optimal solutions for the one satisfying the conditions. The following two theorems give sufficient conditions for exactness of the CQRs and strong Lagrangian duality based on one (any) optimal solution of the CQRs. In fact, these sufficient conditions ensure that there is always an optimal solution to the CQRs $\left(P_{1}\right)$ and $\left(P_{2}\right)$ satisfying the conditions given in Theorems 3.1 and 3.2. Before that, we need the following proposition.

Proposition 3.3 ([37], Lem. 2.5). Suppose that Assumption 2.1 holds. Then $z^{T} B z<0$ for all $z \in \operatorname{Null}\left(A+\lambda_{2} B\right)$ and $z^{T} B z>0$ for all $z \in \operatorname{Null}\left(A+\lambda_{1} B\right)$.

Proof. Let $\hat{\lambda}$ be the same as in Assumption 2.1 and $z \in \operatorname{Null}\left(A+\lambda_{2} B\right)$. We have $A+\hat{\lambda} B=A+\lambda_{2} B+\left(\hat{\lambda}-\lambda_{2}\right) B$. Then

$$
0<z^{T}(A+\hat{\lambda} B) z=z^{T}\left(A+\lambda_{2} B\right) z+\left(\hat{\lambda}-\lambda_{2}\right) z^{T} B z=\left(\hat{\lambda}-\lambda_{2}\right) z^{T} B z .
$$

Since $\hat{\lambda}<\lambda_{2}$, we conclude that $z^{T} B z<0$. Similarly, we have

$$
0<z^{T}(A+\hat{\lambda} B) z=z^{T}\left(A+\lambda_{1} B\right) z+\left(\hat{\lambda}-\lambda_{1}\right) z^{T} B z=\left(\hat{\lambda}-\lambda_{1}\right) z^{T} B z .
$$

Since $\hat{\lambda}>\lambda_{1}$, we conclude that $z^{T} B z>0$.

Theorem 3.4. Suppose that Assumptions 2.1, 2.2 and Condition 1 hold, $\left(x^{*}, t^{*}\right)$ is an optimal solution of problem $\left(P_{1}\right)$ and one of the following holds:

(1) $h_{1}\left(x^{*}\right)=h_{2}\left(x^{*}\right)=t^{*}$.

(2) $h_{1}\left(x^{*}\right)<t^{*}, q_{3}\left(x^{*}\right)<0$ and there exists nonzero $z \in \operatorname{Null}\left(A+\lambda_{2} B\right) \cap \operatorname{Null}(C)$.

(3) $h_{1}\left(x^{*}\right)<t^{*}, q_{3}\left(x^{*}\right)=0$ and there exists nonzero $z \in \operatorname{Null}\left(A+\lambda_{2} B\right) \cap \operatorname{Null}(C)$ such that $\left(a+\lambda_{2} b\right)^{T} z=0$.

(4) $h_{1}\left(x^{*}\right)<t^{*}, q_{3}\left(x^{*}\right)<0$, $\operatorname{Null}\left(A+\lambda_{2} B\right) \cap \operatorname{Null}(C)=\emptyset$ and either $\alpha_{1} \leq \alpha_{1}^{\prime}$ or $\alpha_{2} \geq \alpha_{2}^{\prime}$ where $\alpha_{1}$ and $\alpha_{2}$ are the positive and negative roots of the quadratic equation $q_{2}\left(x^{*}+\alpha z\right)=0$, respectively, $\alpha_{1}^{\prime}$ and $\alpha_{2}^{\prime}$ are the positive and negative roots of the quadratic equation $q_{3}\left(x^{*}+\alpha z\right)=0$, respectively, and $0 \neq z \in \operatorname{Null}\left(A+\lambda_{2} B\right)$.

(5) $h_{2}\left(x^{*}\right)<t^{*}$ and $\hat{\lambda}_{1}=0$.

(6) $h_{2}\left(x^{*}\right)<t^{*}, \hat{\lambda}_{1}>0, q_{3}\left(x^{*}\right)<0$ and there exists nonzero $z \in \operatorname{Null}\left(A+\hat{\lambda}_{1} B\right) \cap \operatorname{Null}(C)$.

(7) $h_{2}\left(x^{*}\right)<t^{*}, \hat{\lambda}_{1}>0, q_{3}\left(x^{*}\right)=0$ and there exists nonzero $z \in \operatorname{Null}\left(A+\hat{\lambda}_{1} B\right) \cap \operatorname{Null}(C)$ such that $\left(a+\hat{\lambda}_{1} b\right)^{T} z=$ 0.

(8) $h_{2}\left(x^{*}\right)<t^{*}, \hat{\lambda}_{1}>0, q_{3}\left(x^{*}\right)<0, \operatorname{Null}\left(A+\hat{\lambda}_{1} B\right) \cap \operatorname{Null}(C)=\emptyset$ and either $\alpha_{1} \leq \alpha_{1}^{\prime}$ or $\alpha_{2} \geq \alpha_{2}^{\prime}$ where $\alpha_{1}$ and $\alpha_{2}$ are the positive and negative roots of the quadratic equation $q_{2}\left(x^{*}+\alpha z\right)=0$, respectively, $\alpha_{1}^{\prime}$ and $\alpha_{2}^{\prime}$ are the positive and negative roots of the quadratic equation $q_{3}\left(x^{*}+\alpha z\right)=0$, respectively, and $0 \neq z \in \operatorname{Null}\left(A+\hat{\lambda}_{1} B\right)$. 
Then problem $(P)$ is equivalent to $\left(P_{1}\right)$, i.e., $p^{*}=p_{1}^{*}$, strong duality holds for $(P)$ and its Lagrangian dual problem is solvable. Also, in cases (1) and (5), $x^{*}$ is an optimal solution to (P) and in cases (2), (3), (6) and (7), $\bar{x}^{*}:=x^{*}+\alpha^{*} z$ is an optimal solution for $(P)$ where $\alpha^{*}$ is the positive root of the quadratic equation $q_{2}\left(x^{*}+\alpha z\right)=0$ and in cases (4) and (8), $\bar{x}^{*}$ solves problem (P) that $\bar{x}^{*}:=x^{*}+\alpha_{1} z$ if $\alpha_{1} \leq \alpha_{1}^{\prime}$ and $\bar{x}^{*}:=x^{*}+\alpha_{2} z$ if $\alpha_{2} \geq \alpha_{2}^{\prime}$.

Proof. (1) It follows from Theorems 3.1 and 3.2.

(2) Consider the optimality conditions (3.2)-(3.9). In this case, $\mu_{2}^{*}=1$ and hence, $h_{2}\left(x^{*}\right)=t^{*}$. Then $h_{1}\left(x^{*}\right)<t^{*}$ and $h_{2}\left(x^{*}\right)=t^{*}$ imply that $\left(\hat{\lambda}_{1}-\lambda_{2}\right) q_{2}\left(x^{*}\right)<0$. Since $\hat{\lambda}_{1}<\lambda_{2}$, we obtain

$$
q_{2}\left(x^{*}\right)>0 .
$$

By the assumption, there exists nonzero $z \in \operatorname{Null}\left(A+\lambda_{2} B\right) \cap \operatorname{Null}(C)$. By replacing $z$ with $-z$ if necessary, we assume without loss of generality that $d^{T} z \leq 0$. Consider the following quadratic equation of variable $\alpha$ :

$$
q_{2}\left(x^{*}+\alpha z\right)=\alpha^{2} z^{T} B z+2 \alpha\left(z^{T} B x^{*}+b^{T} z\right)+q_{2}\left(x^{*}\right)=0 .
$$

By Proposition 3.3, $z^{T} B z<0$. This together with (3.17) imply that the above equation has a positive root $\alpha^{*}$. Moreover, we have

$$
q_{3}\left(x^{*}+\alpha^{*} z\right)=2 d^{T} z \alpha^{*}+q_{3}\left(x^{*}\right)<0,
$$

since $C z=0, d^{T} z \leq 0, \alpha^{*}>0$ and $q_{3}\left(x^{*}\right)<0$. Set $\bar{x}^{*}=x^{*}+\alpha^{*} z$. We have $q_{2}\left(\bar{x}^{*}\right)=0$ and $q_{3}\left(\bar{x}^{*}\right)<0$. Furthermore, since $q_{3}\left(x^{*}\right)<0$, then $\mu_{3}^{*}=0$ and thus relation (3.2) reduces to

$$
\left(A+\lambda_{2} B\right) x^{*}=-\left(a+\lambda_{2} b\right),
$$

implying that $\left(a+\lambda_{2} b\right)^{T} z=0$. This further implies that

$$
h_{2}\left(\bar{x}^{*}\right)=\bar{x}^{*^{T}}\left(A+\lambda_{2} B\right) \bar{x}^{*}+2\left(a+\lambda_{2} b\right)^{T} \bar{x}^{*}+\lambda_{2} \beta=h_{2}\left(x^{*}\right)=t^{*} .
$$

Next, it follows from (3.19) and $q_{2}\left(\bar{x}^{*}\right)=0$ that $q_{1}\left(\bar{x}^{*}\right)=t^{*}$ and consequently $h_{1}\left(\bar{x}^{*}\right)=t^{*}$. The above discussion together with the fact that $\left(A+\lambda_{2} B\right) \bar{x}^{*}=-\left(a+\lambda_{2} b\right)$, indicate that $\left(\bar{x}^{*}, t^{*}\right)$ is an optimal solution of $\left(P_{1}\right)$ for which $h_{1}\left(\bar{x}^{*}\right)=h_{2}\left(\bar{x}^{*}\right)=t^{*}$. Therefore, by Theorems 3.1 and $3.2, p^{*}=p_{1}^{*}, \bar{x}^{*}$ solves $(\mathrm{P})$ and strong Lagrangian duality holds for $(\mathrm{P})$ and the Lagrangian dual problem is solvable.

(3) By the assumption, there exists nonzero $z \in \operatorname{Null}\left(A+\lambda_{2} B\right) \cap \operatorname{Null}(C)$ such that $\left(a+\lambda_{2} b\right)^{T} z=0$. By replacing $z$ with $-z$ if necessary, we assume without loss of generality that $d^{T} z \leq 0$. Similar to part (2), we have $\mu_{2}^{*}=1, h_{2}\left(x^{*}\right)=t^{*}, q_{2}\left(x^{*}\right)>0$ and the following quadratic equation of variable $\alpha$ :

$$
q_{2}\left(x^{*}+\alpha z\right)=\alpha^{2} z^{T} B z+2 \alpha\left(z^{T} B x^{*}+b^{T} z\right)+q_{2}\left(x^{*}\right)=0,
$$

has a positive root $\alpha^{*}$. Moreover, since $C z=0, d^{T} z \leq 0$ and $q_{3}\left(x^{*}\right)=0$, then $q_{3}\left(x^{*}+\alpha^{*} z\right) \leq 0$. Set $\bar{x}^{*}=x^{*}+\alpha^{*} z$. We have $q_{2}\left(\bar{x}^{*}\right)=0$ and $q_{3}\left(\bar{x}^{*}\right) \leq 0$. Furthermore, since $\left(a+\lambda_{2} b\right)^{T} z=0$, then

$$
h_{2}\left(\bar{x}^{*}\right)=\bar{x}^{*^{T}}\left(A+\lambda_{2} B\right) \bar{x}^{*}+2\left(a+\lambda_{2} b\right)^{T} \bar{x}^{*}+\lambda_{2} \beta=h_{2}\left(x^{*}\right)=t^{*} .
$$

Next, it follows from (3.21) and $q_{2}\left(\bar{x}^{*}\right)=0$ that $q_{1}\left(\bar{x}^{*}\right)=t^{*}$ and thus $h_{1}\left(\bar{x}^{*}\right)=t^{*}$. These together with the fact that $\left(A+\lambda_{2} B+\mu_{3}^{*} C\right) \bar{x}^{*}=-\left(a+\lambda_{2} b+\mu_{3}^{*} d\right)$ indicate that $\left(\bar{x}^{*}, t^{*}\right)$ is an optimal solution of $\left(P_{1}\right)$ for which $h_{1}\left(\bar{x}^{*}\right)=h_{2}\left(\bar{x}^{*}\right)=t^{*}$. Therefore, by Theorems 3.1 and $3.2, p^{*}=p_{1}^{*}, \bar{x}^{*}$ solves $(\mathrm{P})$, strong Lagrangian duality holds for $(\mathrm{P})$ and the Lagrangian dual problem is solvable.

(4) In this case, similar to part (2), $\mu_{2}^{*}=1, h_{2}\left(x^{*}\right)=t^{*}$ and $q_{2}\left(x^{*}\right)>0$. Take $z \in \operatorname{Null}\left(A+\lambda_{2} B\right)$ (note that $\left(A+\lambda_{2} B\right)$ is singular) and consider the following quadratic equation of variable $\alpha$ :

$$
q_{2}\left(x^{*}+\alpha z\right)=\alpha^{2} z^{T} B z+2 \alpha\left(z^{T} B x^{*}+b^{T} z\right)+q_{2}\left(x^{*}\right)=0 .
$$


The facts that $z^{T} B z<0$ and $q_{2}\left(x^{*}\right)>0$ imply that the above equation has two roots, namely $\alpha_{1}>0$ and $\alpha_{2}<0$. On the other hand, since $C \succeq 0$ and $z \notin \operatorname{Null}(C), z^{T} C z>0$. This with $q_{3}\left(x^{*}\right)<0$ prove that the quadratic equation of variable $\alpha$ :

$$
q_{3}\left(x^{*}+\alpha z\right)=\alpha^{2} z^{T} C z+2 \alpha\left(z^{T} C x^{*}+d^{T} z\right)+q_{3}\left(x^{*}\right)=0,
$$

has two roots, namely $\alpha_{1}^{\prime}>0$ and $\alpha_{2}^{\prime}<0$. Now let $\alpha_{1} \leq \alpha_{1}^{\prime}$ and set $\bar{x}^{*}=x^{*}+\alpha_{1} z$. Then, obviously $q_{2}\left(\bar{x}^{*}\right)=0$ and $q_{3}\left(\bar{x}^{*}\right) \leq 0$. The same discussion as in part (2) proves that $p^{*}=p_{1}^{*}, \bar{x}^{*}$ solves $(\mathrm{P})$, strong duality holds for $(\mathrm{P})$ and the Lagrangian dual problem is solvable. For the case where $\alpha_{2} \geq \alpha_{2}^{\prime}$, it is easy to see that $\bar{x}^{*}=x^{*}+\alpha_{2} z$ solves problem $(\mathrm{P})$.

(5) It follows from Theorems 3.1 and 3.2.

(6) In this case, it is easy to see that $\mu_{1}^{*}=1, h_{1}\left(x^{*}\right)=t^{*}$ and $q_{2}\left(x^{*}\right)<0$. By the assumption, there exists nonzero $z \in \operatorname{Null}\left(A+\hat{\lambda}_{1} B\right) \cap \operatorname{Null}(C)$. By replacing $z$ with $-z$ if necessary, we assume without loss of generality that $d^{T} z \leq 0$. Consider the following quadratic equation of variable $\alpha$ :

$$
q_{2}\left(x^{*}+\alpha z\right)=\alpha^{2} z^{T} B z+2 \alpha\left(z^{T} B x^{*}+b^{T} z\right)+q_{2}\left(x^{*}\right)=0 .
$$

The fact that $z^{T} B z>0$ (see Prop. 3.3) with $q_{2}\left(x^{*}\right)<0$ imply that the above equation has a positive root $\alpha^{*}$. Moreover, we have

$$
q_{3}\left(x^{*}+\alpha^{*} z\right)=2 d^{T} z \alpha^{*}+q_{3}\left(x^{*}\right)<0
$$

since $C z=0, d^{T} z \leq 0, \alpha^{*}>0$ and $q_{3}\left(x^{*}\right)<0$. Set $\bar{x}^{*}=x^{*}+\alpha^{*} z$. We have $q_{2}\left(\bar{x}^{*}\right)=0$ and $q_{3}\left(\bar{x}^{*}\right)<0$. Furthermore, since $q_{3}\left(x^{*}\right)<0$, then $\mu_{3}^{*}=0$ and thus relation (3.2) reduces to

$$
\left(A+\hat{\lambda}_{1} B\right) x^{*}=-\left(a+\hat{\lambda}_{1} b\right),
$$

implying that $\left(a+\hat{\lambda}_{1} b\right)^{T} z=0$. This further implies that

$$
h_{1}\left(\bar{x}^{*}\right)=\bar{x}^{*^{T}}\left(A+\hat{\lambda}_{1} B\right) \bar{x}^{*}+2\left(a+\hat{\lambda}_{1} b\right)^{T} \bar{x}^{*}+\hat{\lambda}_{1} \beta=h_{1}\left(x^{*}\right)=t^{*} .
$$

Next, it follows from $(3.22)$ and $q_{2}\left(\bar{x}^{*}\right)=0$ that $q_{1}\left(\bar{x}^{*}\right)=t^{*}$ and consequently $h_{2}\left(\bar{x}^{*}\right)=t^{*}$. The above discussion together with the fact that $\left(A+\hat{\lambda}_{1} B\right) \bar{x}^{*}=-\left(a+\hat{\lambda}_{1} b\right)$, indicate that $\left(\bar{x}^{*}, t^{*}\right)$ is an optimal solution of $\left(P_{1}\right)$ for which $h_{1}\left(\bar{x}^{*}\right)=h_{2}\left(\bar{x}^{*}\right)=t^{*}$. Therefore, by Theorems 3.1 and $3.2, p^{*}=p_{1}^{*}, \bar{x}^{*}$ solves $(\mathrm{P})$, strong duality holds for $(\mathrm{P})$ and the Lagrangian dual problem is solvable.

(7) In this case, $\mu_{1}^{*}=1, h_{1}\left(x^{*}\right)=t^{*}$ and $q_{2}\left(x^{*}\right)<0$. Then following the same discussion as in part (3) where $\lambda_{2}$ is replaced by $\hat{\lambda}_{1}$ and $h_{2}\left(\bar{x}^{*}\right)$ in (3.21) is replaced by $h_{1}\left(\bar{x}^{*}\right)$ completes the proof.

(8) In this case, $\mu_{1}^{*}=1, h_{1}\left(x^{*}\right)=t^{*}$ and $q_{2}\left(x^{*}\right)<0$. Then following the same discussion as in part (4) where $\lambda_{2}$ is replaced by $\hat{\lambda}_{1}$ completes the proof.

Theorem 3.5. Suppose that Assumptions 2.1, 2.2 and Condition 2 hold, $x^{*}$ is an optimal solution of problem $\left(P_{2}\right)$ and one of the following holds:

(1) $q_{2}\left(x^{*}\right)=0$.

(2) $q_{2}\left(x^{*}\right)<0, q_{3}\left(x^{*}\right)<0$ and there exists nonzero $z \in \operatorname{Null}\left(A+\hat{\lambda}_{1} B\right) \cap \operatorname{Null}(C)$.

(3) $q_{2}\left(x^{*}\right)<0, q_{3}\left(x^{*}\right)=0$ and there exists nonzero $z \in \operatorname{Null}\left(A+\hat{\lambda}_{1} B\right) \cap \operatorname{Null}(C)$ such that $\left(a+\hat{\lambda}_{1} b\right)^{T} z=0$.

(4) $q_{2}\left(x^{*}\right)<0, q_{3}\left(x^{*}\right)<0$, $\operatorname{Null}\left(A+\hat{\lambda}_{1} B\right) \cap \operatorname{Null}(C)=\emptyset$ and either $\alpha_{1} \leq \alpha_{1}^{\prime}$ or $\alpha_{2} \geq \alpha_{2}^{\prime}$ where $\alpha_{1}$ and $\alpha_{2}$ are the positive and negative roots of the quadratic equation $q_{2}\left(x^{*}+\alpha z\right)=0$, respectively, $\alpha_{1}^{\prime}$ and $\alpha_{2}^{\prime}$ are the positive and negative roots of the quadratic equation $q_{3}\left(x^{*}+\alpha z\right)=0$, respectively, and $0 \neq z \in \operatorname{Null}\left(A+\hat{\lambda}_{1} B\right)$.

Then problem $(P)$ is equivalent to $\left(P_{2}\right)$, i.e., $p^{*}=p_{2}^{*}$, strong duality holds for $(P)$ and its Lagrangian dual problem is solvable. Also, in case (1), $x^{*}$ is an optimal solution to (P), in case (2) and (3), $\bar{x}^{*}:=x^{*}+\alpha^{*} z$ is optimal for $(P)$ where $\alpha^{*}$ is the positive root of the quadratic equation $q_{2}\left(x^{*}+\alpha z\right)=0$ and in case (4), $\bar{x}^{*}$ solves problem $(P)$ that $\bar{x}^{*}:=x^{*}+\alpha_{1} z$ if $\alpha_{1} \leq \alpha_{1}^{\prime}$ and $\bar{x}^{*}:=x^{*}+\alpha_{2} z$ if $\alpha_{2} \geq \alpha_{2}^{\prime}$. 
Proof. (1) It follows from Theorems 3.1 and 3.2.

(2) Consider the optimality conditions (3.12)-(3.16). In this case $\mu_{1}^{*}=0$. By the assumption, there exists nonzero $z \in \operatorname{Null}\left(A+\hat{\lambda}_{1} B\right) \cap \operatorname{Null}(C)$. By replacing $z$ with $-z$ if necessary, we may assume without loss of generality that $d^{T} z \leq 0$. Consider the following quadratic equation of variable $\alpha$ :

$$
q_{2}\left(x^{*}+\alpha z\right)=\alpha^{2} z^{T} B z+2 \alpha\left(z^{T} B x^{*}+b^{T} z\right)+q_{2}\left(x^{*}\right)=0 .
$$

Since we have assumed that $q_{1}(x)$ and $q_{2}(x)$ are not both convex, $\hat{\lambda}_{1}>0$ and consequently $\hat{\lambda}_{1}=\lambda_{1}$. Then by Proposition 3.3, we have $z^{T} B z>0$. This together with $q_{2}\left(x^{*}\right)<0$ imply that the above equation has a positive root $\alpha^{*}$. Moreover, we have

$$
q_{3}\left(x^{*}+\alpha^{*} z\right)=2 d^{T} z \alpha^{*}+q_{3}\left(x^{*}\right)<0,
$$

since $C z=0, d^{T} z \leq 0, \alpha^{*}>0$ and $q_{3}\left(x^{*}\right)<0$. Set $\bar{x}^{*}=x^{*}+\alpha^{*} z$. We have $q_{2}\left(\bar{x}^{*}\right)=0$ and $q_{3}\left(\bar{x}^{*}\right)<0$. Now it is easy to see that optimality conditions of problem $\left(P_{2}\right)$ hold for $\bar{x}^{*}$ with $\mu_{1}^{*}$ and $\mu_{2}^{*}$, i.e., $\bar{x}^{*}$ is an optimal solution of $\left(P_{2}\right)$ for which $q_{2}\left(\bar{x}^{*}\right)=0$. Therefore, by Theorems 3.1 and $3.2, p^{*}=p_{2}^{*}, \bar{x}^{*}$ solves $(\mathrm{P})$ and strong duality holds for $(\mathrm{P})$ and the Lagrangian dual problem is solvable.

(3) By the assumption, there exists nonzero $z \in \operatorname{Null}\left(A+\hat{\lambda}_{1} B\right) \cap \operatorname{Null}(C)$ such that $\left(a+\hat{\lambda}_{1} b\right)^{T} z=0$. By replacing $z$ with $-z$ if necessary, we assume without loss of generality that $d^{T} z \leq 0$. Now consider $\bar{x}^{*}=x^{*}+\alpha^{*} z$ where $\alpha^{*}$ is defined as in part (2). We have $q_{2}\left(\bar{x}^{*}\right)=0$ and $q_{3}\left(\bar{x}^{*}\right) \leq 0$. Moreover, we have

$$
h_{1}\left(\bar{x}^{*}\right)=\bar{x}^{*^{T}}\left(A+\hat{\lambda}_{1} B\right) \bar{x}^{*}+2\left(a+\hat{\lambda}_{1} b\right)^{T} \bar{x}^{*}+\hat{\lambda}_{1} \beta=h_{1}\left(x^{*}\right)=t^{*}
$$

since $z \in \operatorname{Null}\left(A+\hat{\lambda}_{1} B\right)$ and $\left(a+\hat{\lambda}_{1} b\right)^{T} z=0$. This means that $\bar{x}^{*}$ is also optimal for $\left(P_{2}\right)$ for which $q_{2}\left(\bar{x}^{*}\right)=0$. Therefore, by Theorems 3.1 and $3.2, p^{*}=p_{2}^{*}, \bar{x}^{*}$ solves $(\mathrm{P})$ and strong duality holds for $(\mathrm{P})$ and the Lagrangian dual problem is solvable.

(4) In this case, $\mu_{1}^{*}=0$. Take $z \in \operatorname{Null}\left(A+\hat{\lambda}_{1} B\right)$ (note that $\left(A+\hat{\lambda}_{1} B\right)$ is singular) and consider the following quadratic equation of variable $\alpha$ :

$$
q_{2}\left(x^{*}+\alpha z\right)=\alpha^{2} z^{T} B z+2 \alpha\left(z^{T} B x^{*}+b^{T} z\right)+q_{2}\left(x^{*}\right)=0 .
$$

The facts that $z^{T} B z>0$ and $q_{2}\left(x^{*}\right)<0$ imply that the above equation has two roots, namely $\alpha_{1}>0$ and $\alpha_{2}<0$. On the other hand, since $z^{T} C z>0$ and $q_{3}\left(x^{*}\right)<0$, the quadratic equation of variable $\alpha$ :

$$
q_{3}\left(x^{*}+\alpha z\right)=\alpha^{2} z^{T} C z+2 \alpha\left(z^{T} C x^{*}+d^{T} z\right)+q_{3}\left(x^{*}\right)=0,
$$

has two roots, namely $\alpha_{1}^{\prime}>0$ and $\alpha_{2}^{\prime}<0$. Now let $\alpha_{1} \leq \alpha_{1}^{\prime}$ and set $\bar{x}^{*}=x^{*}+\alpha_{1} z$. Then, obviously $q_{2}\left(\bar{x}^{*}\right)=0$ and $q_{3}\left(\bar{x}^{*}\right) \leq 0$. The same discussion as in part (2) proves that $p^{*}=p_{2}^{*}, \bar{x}^{*}$ solves $(\mathrm{P})$, strong duality holds for $(\mathrm{P})$ and the Lagrangian dual problem is solvable. For the case where $\alpha_{2} \geq \alpha_{2}^{\prime}$, it is easy to see that $\bar{x}^{*}=x^{*}+\alpha_{2} z$ solves problem $(\mathrm{P})$.

Remark 3.6. Each of Items (1)-(8) in Theorem 3.4 and (1)-(4) in Theorem 3.5 is sufficient for strong Lagrangian duality and exactness of the CQRs $\left(P_{1}\right)$ and $\left(P_{2}\right)$, respectively. In the following example we illustrate that none of the Items may hold while the CQR is exact. Moreover, we may not be able to give an exact CQR when none of the Items holds.

Example 3.7. Consider the following problem:

$$
\begin{array}{cl}
\min & q_{1}(x):=-x_{1}^{2}+x_{2}^{2} \\
\text { s.t. } & q_{2}(x):=x_{1}^{2}+x_{2}^{2} \leq 1 \\
& q_{3}(x):=x_{1}^{2}+x_{2}^{2}-2 x_{1}+\frac{3}{4} \leq 0 .
\end{array}
$$


The CQR $\left(P_{2}\right)$ of $(3.23)$ is:

$$
\begin{array}{cl}
\min & h_{1}(x):=2 x_{2}^{2}-1 \\
\text { s.t. } & q_{2}(x)=x_{1}^{2}+x_{2}^{2} \leq 1 \\
& q_{3}(x)=x_{1}^{2}+x_{2}^{2}-2 x_{1}+\frac{3}{4} \leq 0 .
\end{array}
$$

The set of optimal solutions of the CQR (3.24) is $X^{*}=\left\{\left(x_{1}^{*}, x_{2}^{*}\right) \mid \frac{1}{2} \leq x_{1}^{*} \leq 1, x_{2}^{*}=0\right\}$. Set $x^{*}=\left(\frac{1}{2}, 0\right)$. It is an optimal solution of (3.23) for which none of Items (1)-(4) in Theorem 3.5 holds. Precisely, $q_{2}\left(x^{*}\right)<0$, $q_{3}\left(x^{*}\right)=0$ and since $C \succ 0$, there is no nozero vector $z \in \operatorname{Null}\left(A+\hat{\lambda}_{1} B\right) \cap \operatorname{Null} C$ such that $\left(a+\hat{\lambda}_{b}\right)^{T} z=0$. The CQR (3.24) is exact since $x^{*}=(1,0)$ is an optimal solution of (3.24) for which $q_{2}\left(x^{*}\right)=0$. Next change $q_{1}(x)$ to $x_{1}^{2}-x_{2}^{2}$. Then the corresponding CQR is

$$
\begin{array}{cl}
\min & h_{1}(x):=2 x_{1}^{2}-1 \\
\text { s.t. } & q_{2}(x)=x_{1}^{2}+x_{2}^{2} \leq 1 \\
& q_{3}(x)=x_{1}^{2}+x_{2}^{2}-2 x_{1}+\frac{3}{4} \leq 0 .
\end{array}
$$

$x^{*}=\left(\frac{1}{2}, 0\right)$ is the unique optimal solution of $(3.25)$ for which none of Items (1)-(4) in Theorem 3.5 hold. Since $x^{*}$ is the unique optimal solution of $(3.25)$ and $q_{2}\left(x^{*}\right)<0$, by Theorem 3.1, the CQR is not exact.

\subsection{How to compute the vector $z$ in Theorems 3.4 and 3.5}

Here we show that the conditions

$$
\exists 0 \neq z \in \operatorname{Null}\left(A+\lambda_{2} B\right) \cap \operatorname{Null}(C),
$$

and

$$
\exists 0 \neq z \in \operatorname{Null}\left(A+\lambda_{2} B\right) \cap \operatorname{Null}(C), \quad \text { such that } \quad\left(a+\lambda_{2} b\right)^{T} z=0,
$$

in Items 2 and 3 of Theorem 3.4 can be verified efficiently via solving an eigenvalue problem. Since both $A+\lambda_{2} B$ and $C$ are positive semidefinite, then condition (3.26) holds if and only if $\lambda_{\min }\left(A+\lambda_{2} B+C\right)=0$ and $z$ is the corresponding eigenvector. Now consider condition (3.27). If $\left(a+\lambda_{2} b\right)=0$, then condition (3.27) reduces to condition (3.26). Let $\left(a+\lambda_{2} b\right) \neq 0$. Then $\left(a+\lambda_{2} b\right)^{T} z=0$ if and only if $z=W y$ for some $y \in \mathbb{R}^{n-1}$ where $W \in \mathbb{R}^{n \times n-1}$ is a basis of $\operatorname{Null}\left(\left(a+\lambda_{2} b\right)^{T}\right)$. Therefore, condition (3.27) holds if and only if $\lambda_{\min }\left(W^{T}\left(A+\lambda_{2} B+C\right) W\right)=0$ and $z=W y^{*}$ where $y^{*}$ is the corresponding eigenvector. A similar discussion holds when $\lambda_{2}$ is replaced by $\hat{\lambda}_{1}$.

\section{New CONDition For EXACT CQRs, SDO-RELAXATIOn AND STRONG DUALity}

We note that although a direct verification of the sufficient conditions in Theorems 3.4 and 3.5 requires a solution of the CQRs, we will show that it is possible to use these conditions in order to find a sufficient condition in terms of the data of the original problem for exactness of the CQRs, strong Lagrangian duality and equivalently for tightness of the SDO-relaxation of problem $(\mathrm{P})$. Recall that we have assumed at least one of $A$ and $B$ is not positive semidefinite. Otherwise, by Assumption 2.2, problem (P) is a convex quadratic optimization problem which satisfies the Slater condition and hence, it enjoys useful features such as strong duality and exact SDO-relaxation. 
The so-called SDO-relaxation of $(\mathrm{P})$ is

$$
\begin{aligned}
p_{r}^{*}:=\min & M \bullet X \\
\text { s.t. } & M_{0} \bullet X \leq 0, \\
& M_{1} \bullet X \leq 0, \\
& I_{0} \bullet X=1, \\
& X \succeq 0,
\end{aligned}
$$

where

$$
M=\left[\begin{array}{cc}
A & a \\
a^{T} & 0
\end{array}\right], M_{0}=\left[\begin{array}{cc}
B & b \\
b^{T} & \beta
\end{array}\right], M_{1}=\left[\begin{array}{cc}
C & d \\
d^{T} & \gamma
\end{array}\right], I_{0}=\left[\begin{array}{cc}
O_{n \times n} & O_{n \times 1} \\
O_{1 \times n} & 1
\end{array}\right]
$$

The dual of $(\mathrm{SDO})$ is

$$
\begin{aligned}
d^{*}:=\max & s \\
\text { s.t. } & M+y_{0} M_{0}+y_{1} M_{1}-s I_{0} \succeq 0, \\
& y_{0}, y_{1} \geq 0,
\end{aligned}
$$

which is also the Lagrangian dual problem of (P). Note that by Assumption 2.1, problem (D-SDO) is strictly feasible and hence, $d^{*}=p_{r}^{*}$. This together with the fact $d^{*} \leq p_{r}^{*} \leq p^{*}$ imply that strong duality holds for $(\mathrm{P})$ if and only if the SDO-relaxation for $(\mathrm{P})$ is exact.

In what follows, we introduce a sufficient condition based on the data of problem $(\mathrm{P})$ that ensures one of Items (1)-(8) in Theorem 3.4 and (1)-(4) in Theorem 3.5 holds. Therefore, it guarantees exactness of the CQRs $\left(P_{1}\right)$ and $\left(P_{2}\right)$ and strong Lagrangian duality.

Condition 3. Consider problem $(\mathrm{P})$. We say that problem $(\mathrm{P})$ satisfies Condition 3 whenever one of the following holds:

(1) Condition 1 holds, $\hat{\lambda}_{1}=0$ and there exists nonzero $z \in \operatorname{Null}\left(A+\lambda_{2} B\right) \cap \operatorname{Null}(C)$ such that $\left(a+\lambda_{2} b\right)^{T} z \leq 0$ and $d^{T} z \leq 0$.

(2) Condition 1 holds, $\hat{\lambda}_{1}>0$ and there exist nonzero $z_{1} \in \operatorname{Null}\left(A+\lambda_{2} B\right) \cap \operatorname{Null}(C)$ and $z_{2} \in \operatorname{Null}\left(A+\hat{\lambda}_{1} B\right) \cap$ $\operatorname{Null}(C)$ such that $\left(a+\lambda_{2} b\right)^{T} z_{1} \leq 0, d^{T} z_{1} \leq 0,\left(a+\hat{\lambda}_{1} b\right)^{T} z_{2} \leq 0$ and $d^{T} z_{2} \leq 0$.

(3) Condition 2 holds and there exists nonzero $z \in \operatorname{Null}\left(A+\hat{\lambda}_{1} B\right) \cap \operatorname{Null}(C)$ such that $\left(a+\hat{\lambda}_{1} b\right)^{T} z \leq 0$ and $d^{T} z \leq 0$.

Theorem 4.1. Suppose that Assumptions 2.1, 2.2 and Condition 3 hold for problem (P). Then the CQRs $\left(P_{1}\right)$ and $\left(P_{2}\right)$ are exact and problem $(P)$ enjoys strong duality and exact $S D O$-relaxation.

Proof. Suppose that Condition 1 holds and let $\left(x^{*}, t^{*}\right)$ be an optimal solution of $\left(P_{1}\right)$. If $h_{1}\left(x^{*}\right)=h_{2}\left(x^{*}\right)=t^{*}$, then by Theorem 3.4, the CQR $\left(P_{1}\right)$ is exact, strong Lagrangian duality holds for problem $(\mathrm{P})$ and the SDOrelaxation is exact. Otherwise, either $h_{1}\left(x^{*}\right)<t^{*}$ or $h_{2}\left(x^{*}\right)<t^{*}$. We show that in both cases, Condition 3 implies the existence of vector $z$ in Theorem 3.4 and thus the assertion holds. First let $h_{1}\left(x^{*}\right)<t^{*}$. If $q_{3}\left(x^{*}\right)<0$, then by Condition 3, Item (2) in Theorem 3.4 holds. Next suppose that $q_{3}\left(x^{*}\right)=0$. We show that, in this case, for all $z \in \operatorname{Null}\left(A+\lambda_{2} B\right) \cap \operatorname{Null}(C)$ satisfying Condition 3, we have $\left(a+\lambda_{2} b\right)^{T} z=0$, implying that Item (3) in Theorem 3.4 holds. To this end, suppose by contradiction that there exists $z \in \operatorname{Null}\left(A+\lambda_{2} B\right) \cap \operatorname{Null}(C)$ such that $\left(a+\lambda_{2} b\right)^{T} z<0$ and $d^{T} z \leq 0$. Set $\bar{x}^{*}=x^{*}+\alpha^{*} z$ where $\alpha^{*}$ is the positive root of equation (3.20). We have $q_{2}\left(\bar{x}^{*}\right)=0, q_{3}\left(\bar{x}^{*}\right) \leq 0$ and since $\left(a+\lambda_{2} b\right)^{T} z<0$,

$$
h_{2}\left(\bar{x}^{*}\right)=\bar{x}^{*^{T}}\left(A+\lambda_{2} B\right) \bar{x}^{*}+2\left(a+\lambda_{2} b\right)^{T} \bar{x}^{*}+\lambda_{2} \beta<h_{2}\left(x^{*}\right)=t^{*} .
$$


On the other hand, since $q_{2}\left(\bar{x}^{*}\right)=0$, we have $\bar{t}:=h_{1}\left(\bar{x}^{*}\right)=h_{2}\left(\bar{x}^{*}\right)<t^{*}$. These mean that $\left(\bar{x}^{*}, \bar{t}\right)$ is a feasible solution of $\left(P_{1}\right)$ with $\bar{t}<t^{*}$ that contradicts the fact that $\left(x^{*}, t^{*}\right)$ is optimal for $\left(P_{1}\right)$. Next, suppose that $h_{2}\left(x^{*}\right)<t^{*}$. If $\hat{\lambda}_{1}=0$, then by Theorem 3.4, the assertion holds. Otherwise, a similar discussion as above proves the existence of vector $z$ in Items (6) and (7) of Theorem 3.4. Similarly, we can prove the existence of vector $z$ in Items (2) and (3) of Theorem 3.5 when Condition 2 holds.

Remark 4.2. Condition 3 can be checked easily by solving a linear programming problem. In fact, condition

$$
\exists 0 \neq z \in \operatorname{Null}\left(A+\lambda_{2} B\right) \cap \operatorname{Null}(C) \text { s.t }\left(a+\lambda_{2} b\right)^{T} z \leq 0 \text { and } d^{T} z \leq 0,
$$

holds if and only if the linear programming problem

$$
\begin{aligned}
\hat{p}:=\min & \left(a+\lambda_{2} b\right)^{T} z \\
\text { s.t. } & \left(A+\lambda_{2} B\right) z=0, \\
& C z=0, \\
& d^{T} z \leq 0,
\end{aligned}
$$

is either unbounded from below or has multiple optimal solutions. If problem (4.2) is unbounded from below, then obviously condition (4.1) holds. If problem (4.2) is bounded, then obviously $\hat{p}=0$. In this case, since $z=0$ is an optimal solution of (4.2), condition (4.1) holds if and only if (4.2) has multiple optimal solutions. The same discussion holds when $\lambda_{2}$ is replaced by $\hat{\lambda}_{1}$.

Remark 4.3. We point out that the sufficient conditions established in Theorems 3.4 and 3.5 are more general than Condition 3. The following is an example where Condition 3 does not hold, while the condition in Theorem 3.5 holds.

Example 4.4. Consider the following problem:

$$
\begin{array}{cl}
\min & q_{1}(x):=-x_{1}^{2}+2 x_{2}^{2} \\
\text { s.t. } & q_{2}(x):=x_{1}^{2}+x_{2}^{2}-1 \leq 0, \\
& q_{3}(x):=x_{1}^{2}+x_{2}^{2}-2 x_{1}-2 x_{2}+1 \leq 0 .
\end{array}
$$

The CQR relaxation of (4.3) is

$$
\begin{array}{cl}
\min & h_{1}(x)=3 x_{2}^{2}-1 \\
\text { s.t. } & q_{2}(x):=x_{1}^{2}+x_{2}^{2}-1 \leq 0, \\
& q_{3}(x):=x_{1}^{2}+x_{2}^{2}-2 x_{1}-2 x_{2}+1 \leq 0 .
\end{array}
$$

The optimal solution of $(4.4)$ is $\left(x_{1}^{*}, x_{2}^{*}\right)=(1,0)$ and $q_{2}\left(x^{*}\right)=0$. Hence, the sufficient condition in Theorem 3.5 is fulfilled. However, Condition 3 is not fulfilled sinc $C=I$ is nonsingular. Moreover, it is easy to verify that strong duality holds for problem (4.3) and the SDO-relaxation and the CQR (4.4) are exact.

\subsection{Comparison with the related conditions from the literature}

Recently, Ho-Nguyen and Kilinç-Karzan [22] have examined variants of TRS having additional conic constraints,

$$
\begin{array}{cl}
\min & q_{1}(x)=x^{T} A x+2 a^{T} x \\
\text { s.t. } & \|x\| \leq 1, \\
& H x-h \in K,
\end{array}
$$


where $A \in \mathbb{R}^{n \times n}$ is a symmetric matrix, $H \in \mathbb{R}^{m \times n}, h \in \mathbb{R}^{m}$ and $K \subseteq \mathbb{R}^{m}$ is a closed convex cone. Assuming $\lambda_{\min }(A)<0$, they proposed the following convex relaxation for (4.5):

$$
\begin{aligned}
\min & q_{1}(x)+\lambda_{\min }(A)\left(1-\|x\|^{2}\right) \\
\text { s.t. } & \|x\| \leq 1, \\
& H x-h \in K,
\end{aligned}
$$

They showed that this convex relaxation is exact if the following condition is satisfied.

Condition 4. There exists nonzero $z \in \operatorname{Null}\left(A-\lambda_{\min }(A) I\right)$ such that $H z \in K$ and $a^{T} z \leq 0$.

Problem (4.5) covers the CDT problem (problem (P) with $B=I, b=0$ and $C \succeq 0$ ) as a special case since the convex quadratic constraint

$$
x^{T} C x+2 d^{T} x+\gamma \leq 0,
$$

can be expressed as the conic constraint $H x-h \in K$ where $K$ is the second-order cone and

$$
H=\left[\begin{array}{c}
-d^{T} \\
C^{\frac{1}{2}} \\
d^{T}
\end{array}\right], \quad h=\left[\begin{array}{c}
\frac{-1+\gamma}{2} \\
O_{n \times n} \\
-\frac{1+\gamma}{2}
\end{array}\right] .
$$

Note that the convex relaxation (4.6) is equal to problem $\left(P_{2}\right)$. We have the following result.

Proposition 4.5. For the CDT problem, Conditions 3 and 4 are equal.

Proof. By Condition 4, there exists nonzero $z \in \operatorname{Null}\left(A-\lambda_{\min }(A) I\right)$ such that $H z \in K$ and $a^{T} z \leq 0$. It follows from $H z \in K$ that

$$
\left\|\left[\begin{array}{c}
C^{\frac{1}{2}} z \\
d^{T} z
\end{array}\right]\right\| \leq-d^{T} z
$$

implying that $d^{T} z \leq 0$ and $z \in \operatorname{Null}(C)$.

Jeyakumar and $\mathrm{Li}$ [27] proved exactness of the SDO-relaxation for the following extended trust-region subproblem (eTRS):

$$
\begin{array}{cl}
\min & x^{T} A x+2 a^{T} x \\
\text { s.t. } & \|x\|^{2}+\beta \leq 0, \\
& c_{i}^{T} x \leq d_{i}, \quad i=1, \ldots, m,
\end{array}
$$

under the dimension condition,

$$
\operatorname{dim}\left(\operatorname{Null}\left(A-\lambda_{\min }(A) I\right)\right) \geq s+1,
$$

where $s=\operatorname{dim}\left(\operatorname{span}\left\{c_{1}, \ldots, c_{m}\right\}\right)$. Later, in [23], the authors proved tightness of the SDO-relaxation of eTRS under the following condition

$$
\operatorname{Rank}\left(\left[A-\lambda_{\min }(A) I \quad c_{1}, \ldots, c_{m}\right]\right) \leq n-1,
$$

which is more general than the dimension condition (DC).

In [22], it has been shown that, in the case of eTRS, Condition 4 generalizes conditions (DC) and (RC). Therefore, by Proposition 4.5, Condition 3 generalizes conditions (DC) and (RC) in the case of eTRS with $m=1$. Jeyakumar and $\mathrm{Li}$ [27] extended the dimension condition (DC) to problem (P) with $B=I$ and $C \succeq 0$ as follows:

$$
\operatorname{dim}\left(\operatorname{Null}\left(A-\lambda_{\min }(A) I\right) \cap \operatorname{Null}(C)\right) \geq 2 .
$$

In this case, Condition 2 holds and $\hat{\lambda}_{1}=-\lambda_{\min }(A)$. The following proposition shows that Condition 3 actually improves condition (EDC). 
Proposition 4.6. For problem $(P)$ with $B=I$ and $C \succeq 0$, Condition 3 is more general than condition (EDC).

Proof. We have

$$
\begin{aligned}
& \operatorname{dim}\left(\operatorname{Null}\left(A-\lambda_{\min }(A) I\right) \cap \operatorname{Null}(C)\right) \geq 2 \\
\Longleftrightarrow & \operatorname{dim}\left(\operatorname{Null}\left(\left[A-\lambda_{\min }(A) I \quad C\right]^{T}\right)\right) \geq 2 \\
\Longleftrightarrow & n-\operatorname{Rank}\left(\left[A-\lambda_{\min }(A) I C\right]\right) \geq 2 \\
\Longleftrightarrow & \operatorname{Rank}\left(\left[A-\lambda_{\min }(A) I C\right]\right) \leq n-2 \\
\Longrightarrow & \operatorname{Rank}\left(\left[A-\lambda_{\min }(A) I \quad C \quad a-\lambda_{\min }(A) b\right]\right) \leq n-1,
\end{aligned}
$$

implying that there exists nonzero $z \in \operatorname{Null}\left(A-\lambda_{\min }(A) I\right) \cap \operatorname{Null}(C)$ such that $\left(a-\lambda_{\min }(A) b\right)^{T} z=0$. If $d^{T} z \leq 0$, then Condition 3 holds. If $d^{T} z>0$, then Condition 3 holds with $z:=-z$.

Clearly, if matrix $C$ is positive definite (nonsingular), then both condition (EDC) and Condition 3 fail. In this case, the sufficient conditions provided in Theorem 3.5 may still hold. Example 4.4 is of such a situation.

We now present necessary and sufficient conditions for global optimality of $(\mathrm{P})$ whenever Condition 3 and Assumptions 2.1 and 2.2 are satisfied.

Corollary 4.7. For $(P)$, suppose that Condition 3, Assumptions 2.1 and 2.2 hold. Let $x^{*}$ be a feasible solution of $(P)$. Then $x^{*}$ is a global minimizer of $(P)$ if and only if there exist nonnegative multipliers $\mu_{1}^{*}$ and $\mu_{2}^{*}$ such that the following conditions hold

$$
\begin{aligned}
\left(A+\mu_{1}^{*} B+\mu_{2}^{*} C\right) x^{*} & =-\left(a+\mu_{1}^{*} b+\mu_{2}^{*} d\right), \\
\mu_{1}^{*} q_{2}\left(x^{*}\right) & =0, \\
\mu_{2}^{*} q_{3}\left(x^{*}\right) & =0 \\
\left(A+\mu_{1}^{*} B+\mu_{2}^{*} C\right) & \succeq 0 .
\end{aligned}
$$

Proof. Let $x^{*}$ be a global minimizer of (P). Recall that by Lemma 4.1, strong duality holds for (P). Suppose that $\left(\mu_{1}^{*}, \mu_{2}^{*}\right)$ is an optimal solution of Lagrangian dual of $(\mathrm{P})$ and $d^{*}$ denotes the dual optimal value. We have

$$
\begin{aligned}
p^{*}=d^{*} & =\min _{x}\left\{q_{1}(x)+\mu_{1}^{*} q_{2}(x)+\mu_{2}^{*} q_{3}\left(x^{*}\right)\right\} \\
& \leq q_{1}\left(x^{*}\right)+\mu_{1}^{*} q_{2}\left(x^{*}\right)+\mu_{2}^{*} q_{3}\left(x^{*}\right) \\
& \leq q_{1}\left(x^{*}\right)=p^{*},
\end{aligned}
$$

where the last inequality follows from $\mu_{i}^{*} \geq 0, i=1,2$ and feasibility of $x^{*}$. We conclude that the two inequalities in this chain hold with equality. Since the inequality in the second line is an equality, we conclude that $x^{*}$ is a minimizer of the minimization problem in the first line. This gives relations (4.7) and (4.10). Moreover, it follows from the last line that $\mu_{1}^{*} q_{2}\left(x^{*}\right)+\mu_{2}^{*} q_{3}\left(x^{*}\right) \leq 0$ which with the fact that each term in this sum is nonpositive, we obtain (4.8) and (4.9). Conversely, suppose that $x^{*}$ satisfies (4.7) to (4.10). We have the following chain of inequalities:

$$
\begin{aligned}
p^{*} \geq d^{*}: & =\max _{\mu_{1}, \mu_{2} \geq 0} \min \left\{q_{1}(x)+\mu_{1} q_{2}(x)+\mu_{2} q_{3}(x)\right\} \\
& \geq \min \left\{q_{1}(x)+\mu_{1}^{*} q_{2}(x)+\mu_{2}^{*} q_{3}(x)\right\} \\
& =q_{1}\left(x^{*}\right)+\mu_{1}^{*} q_{2}\left(x^{*}\right)+\mu_{2}^{*} q_{3}\left(x^{*}\right) \\
& =q_{1}\left(x^{*}\right) \geq p^{*},
\end{aligned}
$$

where the first inequality follows from weak duality property, the second equality follows from (4.7) and (4.10), the third equality follows from (4.8) and (4.9) and the last inequality follows from the primal feasibility of $x^{*}$. Therefore, $q_{1}\left(x^{*}\right)=p^{*}$ and so $x^{*}$ solves $(\mathrm{P})$. 


\section{Conclusions}

We studied problem (P) with positive semidefinite $C$ and under the assumption that $A+\hat{\lambda} B \succ 0$ for some $\hat{\lambda} \geq 0$. We introduced two convex quadratic relaxations (CQRs) corresponding to two different conditions for model problem (P) that minimize a linear objective function over three convex quadratic constraints. We presented sufficient conditions based on an optimal solution of the CQRs under which problem $(\mathrm{P})$ is equivalent to exactly one of the CQRs. We also showed that this equivalence reveals strong Lagrangian duality holds for $(\mathrm{P})$ and consequently problem (P) enjoys exact SDO-relaxation. Furthermore, we derived sufficient conditions based on the data of the problem for exactness of the CQRs, strong Lagrangian duality and equivalently for exactness of the SDO-relaxation. Finally, we presented necessary and sufficient conditions for global optimality of $(\mathrm{P})$ under the new conditions.

Possible topics for future research direction would be the identification of further conditions under which the CQRs are exact (maybe even necessary and sufficient conditions), and of other relaxations which are as simple as $\left(P_{1}\right)$ and $\left(P_{2}\right)$, but exactness holds under more general conditions.

Acknowledgements. The authors are grateful to the referees for valuable comments and suggestions that helped us improve this article. The authors would like to thank Qatar University for the full support under the Grant NCBPQUCP-CAS-2020-1.

\section{REFERENCES}

[1] S. Adachi and Y. Nakatsukasa, Eigenvalue-based algorithm and analysis for nonconvex QCQP with one constraint. Math. Program. 173 (2019) 79-116.

[2] S. Adachi, S. Iwata, Y. Nakatsukasa and A. Takeda, Solving the trust region subproblem by a generalized eigenvalue problem. SIAM J. Optim. 27 (2017) 269-291.

[3] F. Alizadeh and D. Goldfarb, Second-order cone programming. Math. Program. 95 (2003) 3-51.

[4] W. Ai and S. Zhang, Strong duality for the CDT subproblem: a necessary and sufficient condition. SIAM J. Optim. 19 (2009) $1735-1756$.

[5] S. Ansary Karbasy and M. Salahi, Quadratic optimization with two ball constraints. Numer. Algebra Control Optim. 10 (2020) $165-175$.

[6] A. Beck and Y.C. Eldar, Strong duality in nonconvex quadratic optimization with two quadratic constraints. SIAM J. Optim. 17 (2006) 844-860.

[7] A. Ben-Tal and D. den Hertog, Hidden conic quadratic representation of some nonconvex quadratic optimization problems. Math. Program. 143 (2014) 1-29.

[8] A. Ben-Tal and M. Teboulle, Hidden convexity in some nonconvex quadratically constrained quadratic programming. Math. Program. 72 (1996) 51-63.

[9] D. Bienstock, A note on polynomial solvability of the CDT problem. SIAM J. Optim. 26 (2016) $488-498$.

[10] P.T. Boggs and J.W. Tolle, Sequential quadratic programming. Acta Numer. 4 (1995) 1-51.

[11] I.M. Bomze and M.L. Overton, Narrowing the difficulty gap for the Celis-Dennis-Tapia problem. Math. Program. 151 (2015) $459-476$.

[12] I.M. Bomze, V. Jeyakumar and G. Li, Extended trust-region problems with one or two balls: exact copositive and Lagrangian relaxations. J. Global Optim. 71 (2018) 551-569.

[13] M.R. Celis, J.E. Dennis and R.A. Tapia, A trust region strategy for nonlinear equality constrained optimization, edited by P.T. Boggs, R.H. Byrd and R.B. Schnabel. In: Numerical Optimization. SIAM, Philadelphia (1985) 71-82.

[14] X.-D. Chen and Y.-X. Yuan, On local solutions of the Celis-Dennis-Tapia subproblem. SIAM J. Optim. 10 (1999) 359-383.

[15] A.R. Conn, N.I.M. Gould and P.L. Toint, Trust Region Methods. SIAM, Philadelphia, PA (2000).

[16] J.M. Feng, G.X. Lin, R.L. Sheu and Y. Xia, Duality and solutions for quadratic programming over single non-homogeneous quadratic constraint. J. Global Optim. 54 (2012) 275-293.

[17] G.C. Fehmers, L.P.J. Kamp and F.W. Sluijter, An algorithm for quadratic optimization with one quadratic constraint and bounds on the variables. Inverse Prob. 14 (1998) 893-901.

[18] C. Fortin and H. Wolkowicz, The trust region subproblem and semidefinite programming. Optim. Methods Softw. 19 (2004) 41-67.

[19] G.H. Golub and U. Von Matt, Quadratically constrained least squares and quadratic problems. Numer. Math. 59 (1991) 186-197.

[20] N.I. Gould, S. Lucidi, M. Roma and P.L. Toint, Solving the trust-region subproblem using the Lanczos method. SIAM J. Optim. 9 (1999) 504-525. 
[21] M. Grant and S. Boyd, CVX: Matlab software for disciplined convex programming, version 2.1. http://cvxr.com/cvx (2014) 21-57.

[22] N. Ho-Nguyen and F. Kilinç-Karzan, A Second-order cone based approach for solving the trust-region subproblem and its variants. SIAM J. Optim. 27 (2017) 1485-1512.

[23] Y. Hsia and R.L. Sheu, Trust region subproblem with a fixed number of additional linear inequality constraints has polynomial complexity. Preprint arXiv:1312.1398 (2013).

[24] Y. Hsia, G.-X. Lin and R.-L. Sheu, A revisit to quadratic programming with one inequality quadratic constraint via matrix pencil. Pac. J. Optim. 10 (2014) 461-481.

[25] V. Jeyakumar and G. Li, Strong duality in robust convex programming: complete characterizations. SIAM J. Optim. 20 (2010) 3384-3407.

[26] V. Jeyakumar and G. Li, A robust von-Neumann minimax theorem for zero-sum games under bounded payoff uncertainty. Oper. Res. Lett. 39 (2011) 109-114.

[27] V. Jeyakumar and G.Y. Li, Trust-region problems with linear inequality constraints: exact SDP relaxation, global optimality and robust optimization. Math. Program. 147 (2014) 171-206.

[28] R. Jiang and D. Li, Simultaneous diagonalization of matrices and its applications in quadratically constrained quadratic programming, SIAM J. Optim. 26 (2016) 1649-1668.

[29] R. Jiang and D. Li, Novel reformulations and efficient algorithms for the generalized trust region subproblem. SIAM J. Optim. 29 (2019) 1603-1633.

[30] R. Jiang, D. Li and B. Wu, SOCP reformulation for the generalized trust region subproblem via a canonical form of two symmetric matrices. Math. Program. 169 (2018) 531-563.

[31] P. Lancaster and L. Rodman, Canonical forms for hermitian matrix pairs under strict equivalence and congruence. SIAM Rev. 47 (2005) 407-443.

[32] M. Locatelli, Some results for quadratic problems with one or two quadratic constraints. Oper. Res. Lett. 43 (2015) $126-131$.

[33] M. Locatelli, Exactness conditions for an SDP relaxation of the extended trust region problem. Optim. Lett. 10 (2016) $1141-1151$.

[34] J.J. Moré, Generalizations of the trust region problem. Optim. Methods Softw. 2 (1993) 189-209.

[35] J.J. Moré and D.C. Sorensen, Computing a trust region step. SIAM J. Sci. Stat. Comput. 4 (1983) 553-572.

[36] S. Omatu and J.H. Seinfeld, Distributed Parameter Systems: Theory and Applications. Clarendon Press (1989).

[37] T. K. Pong and H. Wolkowicz, The generalized trust region subproblem. Comput. Optim. App. 58 (2014) $273-322$.

[38] M.J.D. Powell and Y. Yuan, A trust region algorithm for equality constrained optimization. Math. Program. 49 (1991) $189-211$.

[39] M. Rojas, S.A. Santos and D.C. Sorensen, A new matrix-free algorithm for the large-scale trust-region subproblem. SIAM J. Optim. 11 (2001) 611-646.

[40] S. Sakaue, Y. Nakatsukasa, A. Takeda and S. Iwata, Solving generalized CDT problems via two-parameter eigenvalues. SIAM J. Optim. 26 (2016) 1669-1694.

[41] M. Salahi and A. Taati, An efficient algorithm for solving the generalized trust region subproblem. Comput. Appl. Math. 37 (2018) 395-413.

[42] M. Salahi and A. Taati, A fast eigenvalue approach for solving the trust region subproblem with an additional linear inequality. Comput. Appl. Math. 37 (2018) 329-347.

[43] M. Salahi, A. Taati and H. Wolkowicz, Local nonglobal minima for solving large-scale extended trust-region subproblems. Comput. Optim. App. 66 (2017) 223-244.

[44] J.F. Sturm and S. Zhang, On cones of nonnegative quadratic functions. Math. Oper. Res. 28 (2003) $246-267$.

[45] A. Taati and M. Salahi, A conjugate gradient-based algorithm for large-scale quadratic programming problem with one quadratic constraint. Comput. Optim. App. 74 (2019) 195-223.

[46] A. Taati and M. Salahi, On local non-global minimizers of quadratic optimization problem with a single quadratic constraint. Numer. Funct. Anal. Optim. 41 (2020) 969-1005.

[47] A.L. Wang and F. Kilinç-Karzan, On the tightness of SDP relaxations of QCQPs. Technical Report, arXiv:1911.09195 (2019).

[48] Y. Ye and S. Zhang, New results on quadratic minimization. SIAM J. Optim. 14 (2003) 245-267.

[49] Y. Yuan, On a subproblem of trust region algorithms for constrained optimization. Math. Program. 47 (1990) $53-63$.

[50] Y. Yuan, A dual algorithm for minimizing a quadratic function with two quadratic constraints. J. Comput. Math. 9 (1991) 348-359.

[51] Y. Zhang, Computing a Celis-Dennis-Tapia trust-region step for equality constrained optimization. Math. Program. 55 (1992) $109-124$. 